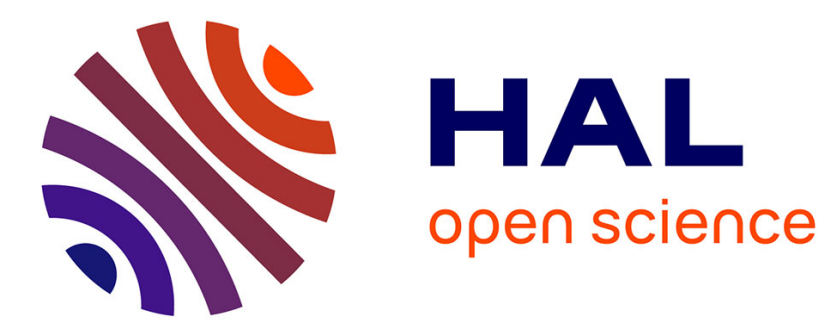

\title{
La période strasbourgeoise de Pierre Montet (1919-1948)
}

Cassandre Hartenstein

\section{To cite this version:}

Cassandre Hartenstein. La période strasbourgeoise de Pierre Montet (1919-1948). Archimède: archéologie et histoire ancienne, 2020, Archimède. Archéologie et histoire ancienne, 8, pp.218-232. 10.47245/archimede.0008.var.03 . halshs-03280152

\section{HAL Id: halshs-03280152 \\ https://shs.hal.science/halshs-03280152}

Submitted on 7 Jul 2021

HAL is a multi-disciplinary open access archive for the deposit and dissemination of scientific research documents, whether they are published or not. The documents may come from teaching and research institutions in France or abroad, or from public or private research centers.
L'archive ouverte pluridisciplinaire HAL, est destinée au dépôt et à la diffusion de documents scientifiques de niveau recherche, publiés ou non, émanant des établissements d'enseignement et de recherche français ou étrangers, des laboratoires publics ou privés. 
1 DOSSIER THÉMATIQUE 1

SIUE DEUS SIUE DEA. DÉNOMINATIONS DIVINES DANS LES MONDES GREC ET SÉMITIQUE : UNE APPROCHE PAR LE GENRE

98 DOSSIER THÉMATIQUE 2

TRADITION ET TRANSMISSION DANS L'ANTIQUITÉ : RÉFLEXIONS INTERDISCIPLINAIRES

159 ACTUALITÉ DE LA RECHERCHE

QUOI DE NEUF À L'OUEST DE STRASBOURG ? KOENIGSHOFFEN :

ÉTAT DES LIEUX ET DÉCOUVERTES RÉCENTES

VARIA

195 Luca BASILE

Forme di contatto, scambio ed interazione culturale nella tradizione vascolare campana tra VIII e VII secolo a.C.

207 Véronique PIтcHoN

Le faste de la table dans la poésie abbasside

\section{Cassandre HARTENSTEIN}

La période strasbourgeoise de Pierre Montet (1919-1948)

233 Anna Maria Desiderio et Arianna Esposito

Genre et mobilité à l'aune des relations socio-culturelles : I'exemple de la Campanie archaïque 


\title{
LA PÉRIODE STRASBOURGEOISE DE PIERRE MONTET (1919-1948)
}

\author{
Cassandre HARTENSTEIN \\ Doctorante \\ Université de Strasbourg \\ UMR 7044 Archimède \\ chartenstein@unistra.fr
}

\section{RÉSUMÉ}

Les acteurs de la refondation de l'Université de Strasbourg en 1919 eurent pour ambition de constituer un corps professoral de haut niveau, pour rivaliser avec la réputation qu'avait acquise la Kaiser-Wilhelms-Universität Straßburg et c'est dans ce contexte que Pierre Montet fut recruté. Ses premières années à Strasbourg confirmèrent son statut de jeune égyptologue prometteur et ses fouilles des nécropoles royales à Byblos, puis à Tanis le firent connaître tant au niveau national qu'international. Cette étude s'appuie sur le dossier du personnel de I'université, qui contient notamment un ensemble de documents concernant la nomination de Pierre Montet et plus particulièrement une lettre de soutien de Victor Loret présentant le vivier des candidats disponibles, selon lui, pour occuper le poste d'enseignant en égyptologie. D'autres archives permettent d'en apprendre plus sur les activités de recherche et d'enseignement de Pierre Montet, de son arrivée à Strasbourg jusqu'à son départ pour le Collège de France, en passant par Clermont-Ferrand où I'université s'était repliée en 1939.
The actors of the rebuilding of the University of Strasbourg in 1919 aimed at forming a high-level corps of professors, with the purpose to compete with the reputation held by Kaiser-Wilhelms-Universität Straßburg and Pierre Montet was recruited in this context. His first years in Strasbourg confirmed that he was a young promising Egyptologist and his excavations in the royal necropoleis in Byblos, then in Tanis made him famous internationally. This paper is based on the human resources department file of Pierre Montet, which contains several pieces about his nomination and especially a support letter of Victor Loret, presenting all the available candidates for the position. Other documents show more information about Pierre Montet's teaching and researching activities, from his arrival in Strasbourg to his leaving for the Collège de France and the period he stayed in Clermond-Ferrand, where the University of Strasbourg was installed since 1939 to 1945

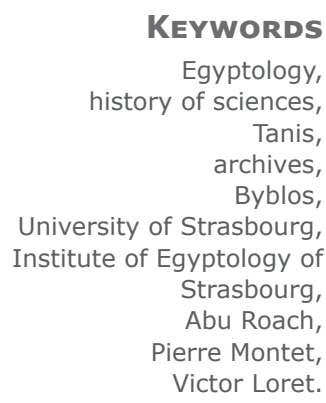


Dès 1915, les autorités françaises discutent du retour de I'Alsace à la France [1], y compris de celui de I'Université de Strasbourg, pour laquelle le Comité de Haute-Alsace a un double objectif : rivaliser avec l'Allemagne en faisant de I'institution alsacienne un modèle de science à la française et amorcer de l'intérieur une réforme des universités, en accordant une place plus importante à la recherche, sans négliger leur mission principale, qui consiste à former des enseignants. À ces deux desseins, les acteurs du renouveau de l'université alsacienne ajoutent l'objectif d'asseoir un rayonnement international de la science alsacienne. La première étape de ce projet ambitieux comprend le limogeage du personnel allemand et la dissolution de la Kaiser-Wilhelms-Universität, qui disparaît officiellement le 7 décembre 1918 [2]. Il faut donc ensuite recruter de nouveaux enseignants, car 104 chaires et 74 maîtrises de conférences sont à pourvoir [3] (ces chiffres ayant pour but principal de surpasser l'ancienne structure allemande, car la comparaison avec I'Universität est un enjeu constant de la reconstruction d'après-guerre). Le recrutement se fait au compte-goutte (en partie parce que bon nombre de candidats sont encore mobilisés), mais presque tous les postes sont pourvus lors de la première rentrée universitaire le 22 novembre 1919.

Discipline phare de la Kaiser-Wilhelms-Universität, l'égyptologie, dont la chaire a été créée en 1872, a acquis une renommée internationale notamment grâce à son deuxième titulaire, W. Spiegelberg, un des plus grands égyptologues du XXe siècle [4]. Lorsque W. Spiegelberg remet les clés de son institut le 27 décembre 1918 avant de quitter définitivement Strasbourg [5], il laisse à son futur successeur un Seminar en excellent état (à l'inverse, certains enseignants français connaissent une grande déception en arrivant à Strasbourg, lorsqu'ils découvrent d'importantes lacunes dans leurs bibliothèques ou dans les équipements laissés par leurs prédécesseurs [6]). Le Seminar d'égyptologie comprend notamment une collection d'antiquités égyptiennes, constituée par W. Spiegelberg à partir de dons, d'achats sur le marché des antiquités et d'artefacts trouvés en fouilles et qui compte 1984 pièces. Créée dans le but de servir de

[1] Olivier-Utard 2010

[2] ID. 2015 , p. $41-42$

[3] ID. 2015, p. 46-47.

[4] Colin 2010 ; Gertzen 2016 ; Voss \& Gertzen 2020.

[5] Archives Départementales du Bas-Rhin (ADBR)

1161 W73, récapitulatif de l'état des collections (objets,

livres) de l'Institut d'égyptologie daté du 27 décembre 1918, support pratique à l'enseignement (tout comme la collection de moulages antiques développée par A. Michaelis pour le Kunstsintitut), elle constitue également un des principaux axes de recherche de W. Spiegelberg, qui a publié un certain nombre de ces objets [7]. En plus de cet élément attractif, I'institut d'égyptologie possède une riche bibliothèque, dont $P$. Montet dira en 1926 : « La bibliothèque égyptologique installée dans la salle 45 où sont réunis en outre les ouvrages de philologie sémitique et de linguistique générale doit sa valeur aux collections fondamentales épuisées aujourd'hui [...]. Tout cela est rassemblé dans une même salle, ouverte à toutes heures du jour, tandis qu'à Lyon, du temps où j'étais étudiant, la bibliothèque du Séminaire ne comptait guère qu'une centaine de volumes et qu'il fallait faire entrer dans la mémoire ce que possédaient quatre ou cinq bibliothèques fort éloignées les unes des autres et leurs heures d'ouverture. À Paris, la difficulté est la même. Il faut aller au Caire, dans la bibliothèque de I'Institut français, pour trouver une plus riche collection d'ouvrages d'égyptologie, aussi aisément accessible » [8]. À ces deux atouts, s'ajoutent les avantages proposés par I'Université de Strasbourg pour attirer des candidats : un salaire supérieur de $25 \%$ à ceux des autres universités de province (les salaires étaient relevés afin d'attirer les enseignants parisiens qui ainsi ne verraient pas leurs revenus diminués), des moyens financiers exceptionnels obtenus grâce à un mécénat productif et un outil d'appui à la recherche, la Bibliothèque Nationale Universitaire [9]. L'étude d'un ensemble de documents aux Archives départementales du Bas-Rhin, entourant la nomination de P. Montet, est I'occasion de jeter un éclairage sur la création de la première chaire d'égyptologie française de I'Université de Strasbourg, dans un contexte historique exceptionnel. Les autres pièces issues du dossier du personnel de P. Montet permettront d'évoquer sa carrière scientifique et son évolution au sein de l'université alsacienne et seront présentées dans les deux dernières parties de cet article, qui correspondent chacune à deux temps forts de l'histoire de l'université strasbourgeoise et de la carrière de $P$. Montet, simultanément.

par W. Spiegelberg.

[6] MüLLER 2018.

[7] SPIEGelberg $1902 ; 1904 ; 1906 ; 1908 ; 1908-1909$;

$1909 ; 1910 ; 1912 ; 1914 ; 1916 ; 1917 ; 1918 a$ et $b ; 1920$.

[8] Montet 1926. Sur la bibliothèque de l'Institut d'égyptologie, voir également LORENTZ 2000, p. 22-24.

[9] MÜLLER 2018. 


\section{LE RECRUTEMENT (1919)}

Il est probable que plusieurs candidats ont postulé à la maîtrise de conférence d'égyptologie strasbourgeoise, puisque $V$. Loret les évoque dans une lettre de recommandation (voir ci-dessous) [10]; mes recherches aux Archives départementales du Bas-Rhin ne m'ont pas permis d'en trouver la trace. Les textes présentés et discutés dans cette étude sont tous issus du dossier du personnel au nom de Pierre Montet [11], ainsi que des archives des doyens de la Faculté des Lettres de I'Université de Strasbourg [12].

Après des études d'égyptologie à I'Université de Lyon, Pierre Montet est entré en tant que pensionnaire à I'Institut Français d'Archéologie Orientale du Caire (Ifao) en 1910 [13]. Il se voit alors confier la responsabilité des fouilles d'Abou Roach près du Caire, par le directeur de l'Ifao, Émile Chassinat, ce qui constitue sa première expérience de terrain en Égypte [14]. P. Montet reste quatre ans en poste au Caire, avant d'être mobilisé en France, où il participe à la Première Guerre mondiale [15]. Il est ensuite affecté le 21 janvier 1918 dans l'armée d'Orient, avant d'être démobilisé le 4 mars 1919.

Dans une lettre rédigée depuis sa résidence de Blacé (dans le Rhône) et datée du 22 mars 1919 [16], Pierre Montet informe le recteur de I'Université de Strasbourg qu'il souhaitait déposer sa candidature pour le poste d'égyptologie dès janvier, mais n'avait pas pu le faire à ce moment, car il était encore mobilisé [17]. Dans cette missive, P. Montet précise qu'il s'apprête à partir pour une mission de trois mois au Liban et en Syrie afin d'effectuer un état des lieux des sites et biens archéologiques. Même si cette déclaration de candidature est bien arrivée à I'université, puisqu'elle est conservée dans les archives, il semblerait que le dossier de P. Montet n'ait pas été pris en compte dans un premier temps par les superviseurs du recrutement. En effet,
P. Montet envoie une seconde lettre de candidature quelques mois plus tard, le 6 septembre [18], depuis Paris, à nouveau à l'intention du recteur. Il s'excuse de transmettre sa candidature si tardivement (il est rentré de sa mission au Moyen-Orient fin août), comme si la première lettre n'était pas arrivée à destination. À la différence de la précédente, $P$. Montet présente dans celle-ci son parcours universitaire, ses travaux et publications et annonce les cours qu'il pourrait donner s'il était nommé.

En parallèle de sa propre démarche, Pierre Montet a demandé à son ancien professeur d'égyptologie de I'Université de Lyon, Victor Loret, de lui écrire une lettre de recommandation [19]. Les archives de l'université ont conservé la trace de l'intervention de V. Loret : dans une première lettre, il écrit au doyen de la Faculté des Lettres (Christian Pfister) pour lui demander l'adresse et la confirmation du nom du nouveau recteur de l'université, afin de lui adresser, comme le lui demande Pierre Montet, une lettre de recommandation. Une petite annotation dans la marge du document, de la main de Chr. Pfister, montre qu'il n'était pas au courant de la candidature de P. Montet [20]. Le second document, la lettre de recommandation [21] (annexe 1 et fig. 1 à 6), présente une rhétorique intéressante : Victor Loret, avec une grande politesse, explique au recteur qu'il va lui donner son opinion (qui n'a pas été demandée) sur tous les candidats éventuels au poste d'égyptologie. Le premier d'entre eux est Raymond Weil, dont le principal défaut, d'après V. Loret, est de ne pas connaitre l'égyptien ancien : « (...) Son grand, son terrible défaut est celui-ci : il ne connaît pas un traître mot d'égyptien ! La grammaire égyptienne lui est chose totalement inconnue. Et le pire est qu'il ne se doute même pas qu'il ne connait pas l'égyptien. De sorte qu'il publie, en toute innocence, les traductions les plus saugrenues et les plus inimaginables, qui font se pâmer de rire, ou d'indignation, selon les
[10] ADBR 1007W 1251, lettre de Victor Loret du 19 septembre 1919 à Sébastien Charléty (directeur général de I'Instruction publique et des Beaux-Arts).

[11] ADBR 1007W 1251.

[12] Les textes de ce dossier seront transcrits et présentés sur le carnet hypothèse de la collection de l'Institut d'égyptologie de Strasbourg : Recollecta Aegyptiaca (https://recaeg. hypotheses.org).

[13] HARTENSTEIN 2017.

[14] WolfF 1967 ; WaLle 1967 ; HelCK 1968-1969 SAUNERON 1968.

[15] Une fiche de renseignement conservée dans le dossier des ressources humaines nous apprend que Pierre Montet a été « récupéré » par l'infanterie armée le 15 février 1915, qu'il a été blessé et a reçu deux citations, en plus de la croix de guerre. Pour plus d'informations sur le parcours de l'égyptologue durant la Première Guerre mondiale, voir ROBERT 2018. Un second document, rédigé de la main de P. Montet, également conservé dans son dossier du personnel, détaille sa carrière militaire, en plus du livret découvert par $B$. Robert.

[16] ADBR 1007W 1251, lettre de P. Montet du 22 mars 1919 adressée au recteur $\mathrm{S}$. Charléty.

[17] ROBERT 2018, p. 16.

[18] ADBR 1007W 1251, lettre de P. Montet du 9 septembre 1919 adressée au recteur S. Charléty. N'étant pas certain que cette lettre parviendrait à son destinataire, $\mathrm{P}$. Montet envoie une troisième lettre de candidature le 10 septembre au recteur, à une adresse parisienne.

[19] ADBR 1007W 1251, lettre de V. Loret du 6 août 1919 au doyen de la Faculté des Lettres Christian Pfister.

[20] ADBR 1007W 1251, lettre de V. Loret au recteur de I'Université de Strasbourg, datée du 9 septembre 1919. [21] ADBR 1007W 1251, lettre de V. Loret au recteur de I'Université de Strasbourg, datée du 9 septembre 1919. 


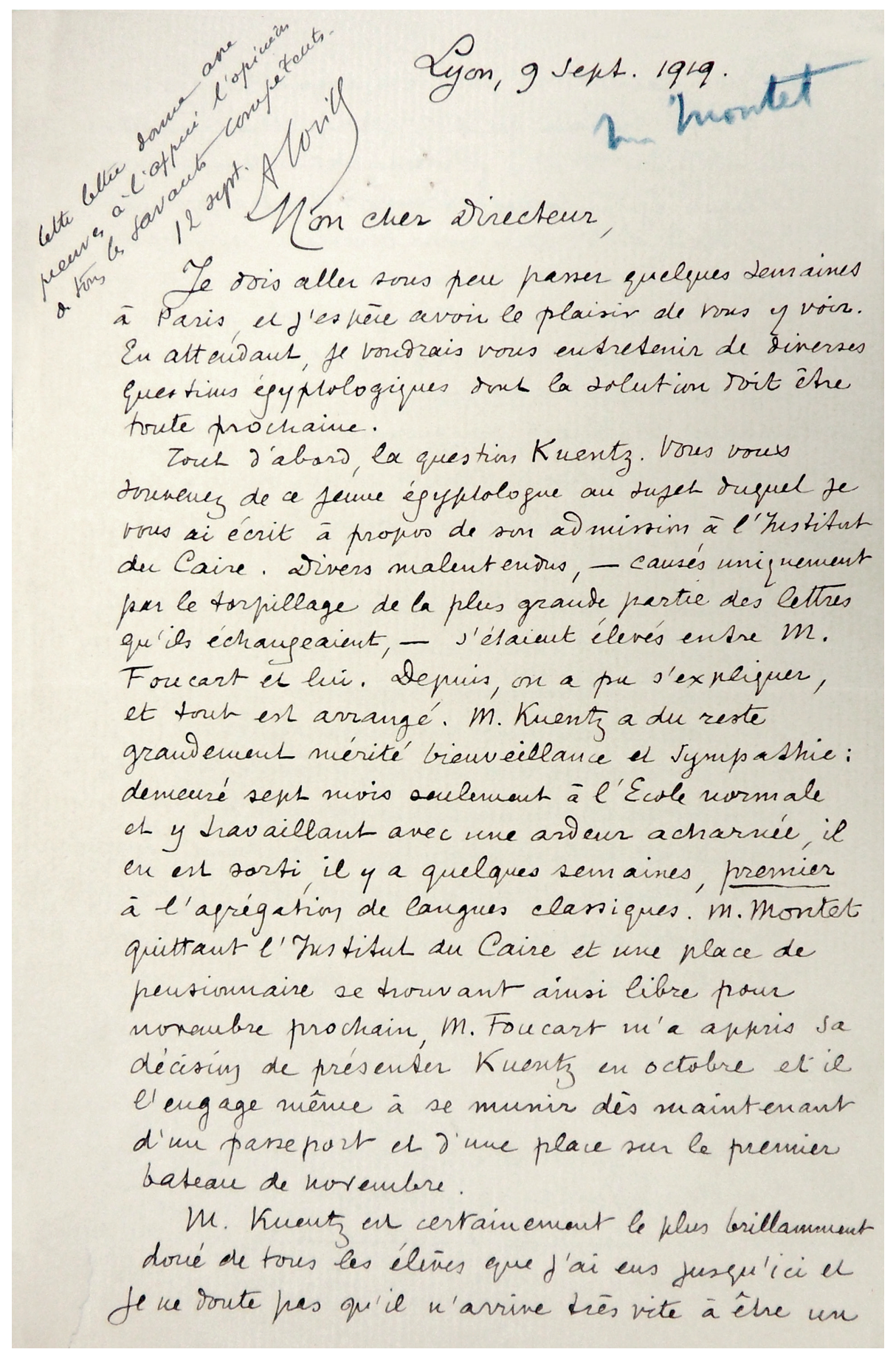

Fig. 1 :

ADBR 1007W 1251,

lettre de V. Loret au recteur

S. Charléty, page 1 .

caractères, ceux qui lisent ses interprétations de textes hiéroglyphiques. (...) Mais lui confier l'enseignement de l'égyptien à Strasbourg ! L'égyptologie allemande en trépignerait de joie ». On notera que V. Loret touche ici un point sensible, en jouant sur la rivalité que la nouvelle université française entretient avec sa devancière et plus généralement, avec l'institution universitaire allemande. Ce jugement à l'encontre de R. Weil peut paraître sévère, surtout lorsqu'on sait qu'il est nommé en cette même année 1919 Directeur d'Études à la Section des Sciences historiques et philologiques de I'École pratique des Hautes Études [22]. Le second candidat à être passé au crible est Jules Baillet, à qui
Victor Loret reproche de ne pas avoir la stature nécessaire pour le poste strasbourgeois : « D'ailleurs, la linguistique orientale n'est pas son fort, loin de là, et il n'a pas dû lire et traduire beaucoup de textes égyptiens, ni surtout avec toute la minutie et la précision qu'il faut y mettre pour en tirer des idées nouvelles. De là, sans doute, ce quelque chose de grisâtre, d'impersonnel, de vieillot, qui se dégage de la lecture de ses écrits. En somme, il n'a, à aucun degré la mentalité d'un professeur d'enseignement supérieur et,

[22] Stracmans 1951 et Vandier 1951. 


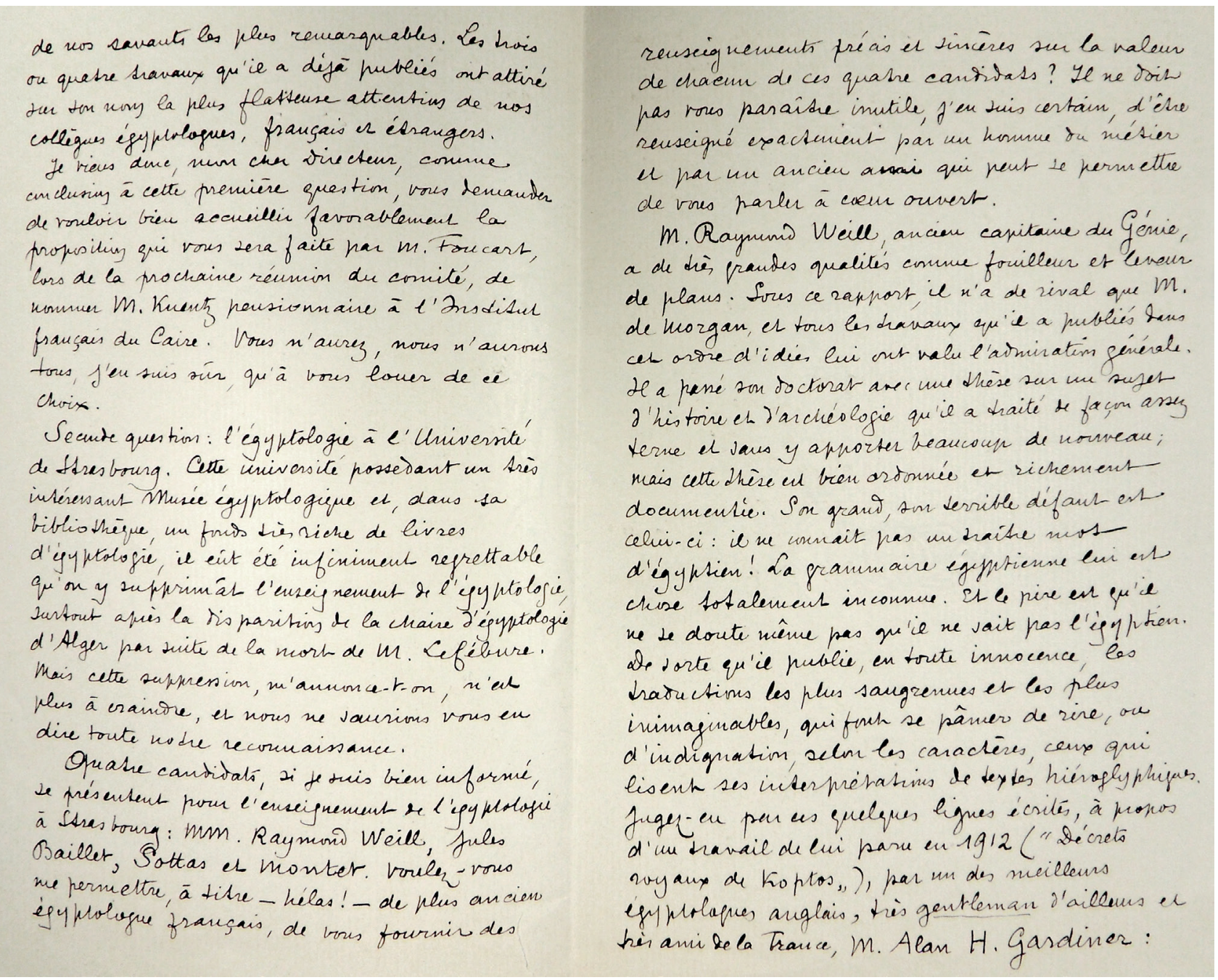

Fig. 2 : ADBR 1007W 1251, lettre de V. Loret au recteur S. Charléty, page 2 et 3.

très vraisemblablement, ne l'acquerra jamais ». Le troisième candidat potentiel est Henri Sottas, qui ne semble pas à première vue affublé de gros défauts scientifiques, car V. Loret le qualifie de prometteur. Néanmoins, il possède « une tare, et même une tare très grave : il n'a jamais mis les pieds en Égypte ! Et cela se voit, dès les premières lignes, dans tout ce qu'il écrit ». Pourtant, dans une note biographique et bibliographique consacrée à Henri Sottas à la suite de son décès et parue en 1927 dans l'Annuaire de I'École Pratique des Hautes Études [23], Raymond Weil, candidat malheureux au poste strasbourgeois, écrit : «Au printemps de cette année 1913, Sottas était parti pour son « tour d'Égypte», un instructif voyage sur les grands sites archéologiques et les champs de fouilles en activité et à l'auteur des présentes notes (donc Raymond Weil), en chantier dans la montagne de Zaouet el-Maietîn, avait rendu visite ». Henri Sottas (qui sera également nommé à l'École pratique des Hautes Études en 1921) est donc éliminé à son tour et permet à Victor Loret d'introduire et d'enchaîner sur le dernier candidat éventuel, un certain Pierre Montet, qui, lui, « a passé par l'Institut du Caire. Il a parcouru le pays dans tous les sens, a même traversé le désert entre le Nil et la mer Rouge (...) ». Après avoir chaleureusement décrit les qualités et donné un aperçu des travaux de Pierre Montet, Victor Loret termine sa lettre ainsi : « Je n'ai pas besoin de vous dire ma conclusion ».

Pierre Montet est nommé officiellement chargé de cours [24] le $1^{\text {er }}$ octobre 1919 et est présent pour la première rentrée de la nouvelle Université de Strasbourg. Pour trouver de nouveaux enseignants, I'université alsacienne se tourne donc, en ce qui concerne I'histoire et I'archéologie, vers le réseau des Instituts français à l'étranger. En tout, trois pensionnaires issus de ces institutions sont recrutés : Pierre Montet, Eugène Cavaignac et Paul Collomp [25].

\section{[23] Weil 1929.}

[24] N'ayant pas encore soutenu sa thèse au moment de son recrutement, Pierre Montet n'est pas nommé maître de conférences, mais chargé de cours.

[25] Olivier-Utard, 2015, p. 65. 
re It is requettable that the broad principles of Egyptian grammar, now to fully and certainly established, strould still be iquored by so active and intus trious an Egyptologist as $m$. Weill. It is difficult to imagine how, ex.g2. The renierings "..." could be arrived at, ani a similar guessing at the sanse is everywhere apparent. As exemples of particularly crass granumatical blunders I will only quote the trauslation ".... etc, ete (Proceidings of the Society of Biblical Ascheology, vol. $x \times x 1 v, L$ London, 1912, p.265).

m. Lacau songerait, m'a.t.on dit, à faire entres $m$. Weill an Service des Ansiquités de l'Egppte, connue successan de Cegrain à la brrection des fowiles de Thibes. Là senait sa vaie nlace et il y renorait o'inmenses services. Thais hi cnefier l'euseynement de l'égefpkien à Stras bourg?

Q'égey ptologie allene ante en drépignesait de foies. m. Jules Baillet ex \& fils s'un egy pologue. Auguste Bailler, rnc les veurres, qui forment deux assey gros nolumes, ons été publicés en collabosation par le piere et le fils el sant quelqueforis, par les es prito tuperficiels, cueforions arec los ouvres du fils. Celles-ci sour peu nombreuses: Lrvis on quare articles (exactement conume $m$. Kuentz) er lewe thises de roctarat, Duch la principale a 810 pages. In. J. Baillet en de puis longtenps profersen de bycie. Thalgré ses accaparantes occupations profersioncles, il a trouné le neryen de se tenir itonnanneut au courants de toute la littérative

Fig. 3 : ADBR 1007W 1251,

lettre de V. Loret au recteur S. Charléty, page 4.

\section{LES PREMIÈRES ANNÉES ET LES FOUILLES A BYBLOS: LE «STATUT TRANSITOIRE 》DE L'UNIVERSITE DE STRASBOURG (1920-1925)}

Les enseignements que Pierre Montet dispense font partie de ce qu'on appelle la licence « libre » ; I'université française après la guerre a principalement vocation à former les maîtres d'école et les professeurs du secondaire qui suivent les cours de la licence « normale». La licence libre forme des chercheurs et des étudiants souhaitant élargir leur culture, mais ce parcours attire moins de candidats. Chaque étudiant peut choisir quatre disciplines de son choix. Lors de son arrivée en 1919, P. Montet dispense trois cours d'une heure par semaine, ce chiffre passant à cinq à partir de l'année suivante. Dans une lettre adressée au doyen de la Faculté des Lettres en 1923 [26], l'égyptologue décrit les cours qu'il donne : « J'ai fait chaque semaine 5 cours ou leçons : Grammaire - explication de textes (réservée à un spécialiste, M. I'abbé Bucher) - Mœurs et coutumes des
Égyptiens - Histoire de l'Art égyptien (pour les candidats à l'agrégation d'histoire qui avaient cette question à leur programme) - Travaux pratiques (au Musée) - ». Le nombre d'étudiants est modeste : 4 élèves et 6 auditeurs en 1919-1920 [27]; seulement 2 inscrits et 5 auditeurs en 1920-1921 [28]. Ces nombres sont comparables aux effectifs également limités du public égyptologique à la fin de la période allemande, connus grâce aux rapports d'activité annuels. En 1913, par exemple, W. Spiegelberg signalait que « dans I'Institut d'égyptologie, pendant l'année académique écoulée, 6 messieurs ont travaillé, dont 5 ont participé aux exercices d'archéologie qui ont eu lieu dans la collection de l'institut » [29].

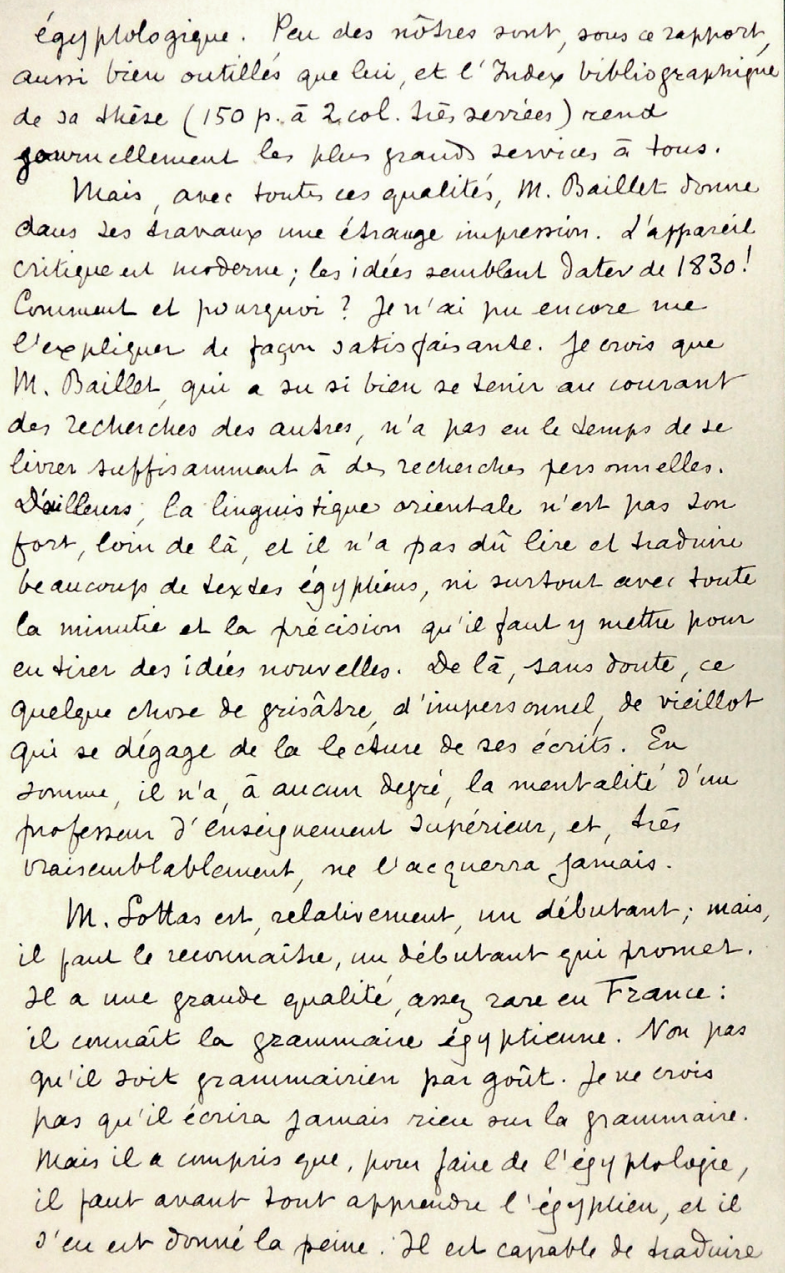

Fig. 4 : ADBR 1007W 1251,

lettre de V. Loret au recteur S. Charléty, page 5.

[26] ADBR 1007W 1251, lettre de P. Montet datée du 15 juin 1923, adressée au doyen Chr. Pfister.

[27] Idem, notice individuelle du Service de I'Instruction publique du 17 mars 1920.

[28] Idem, notice individuelle du Service de l'Instruction publique du 8 juin 1921.

[29] Colin \& Hartenstein 2017, p. 262. 


\begin{tabular}{|c|c|}
\hline 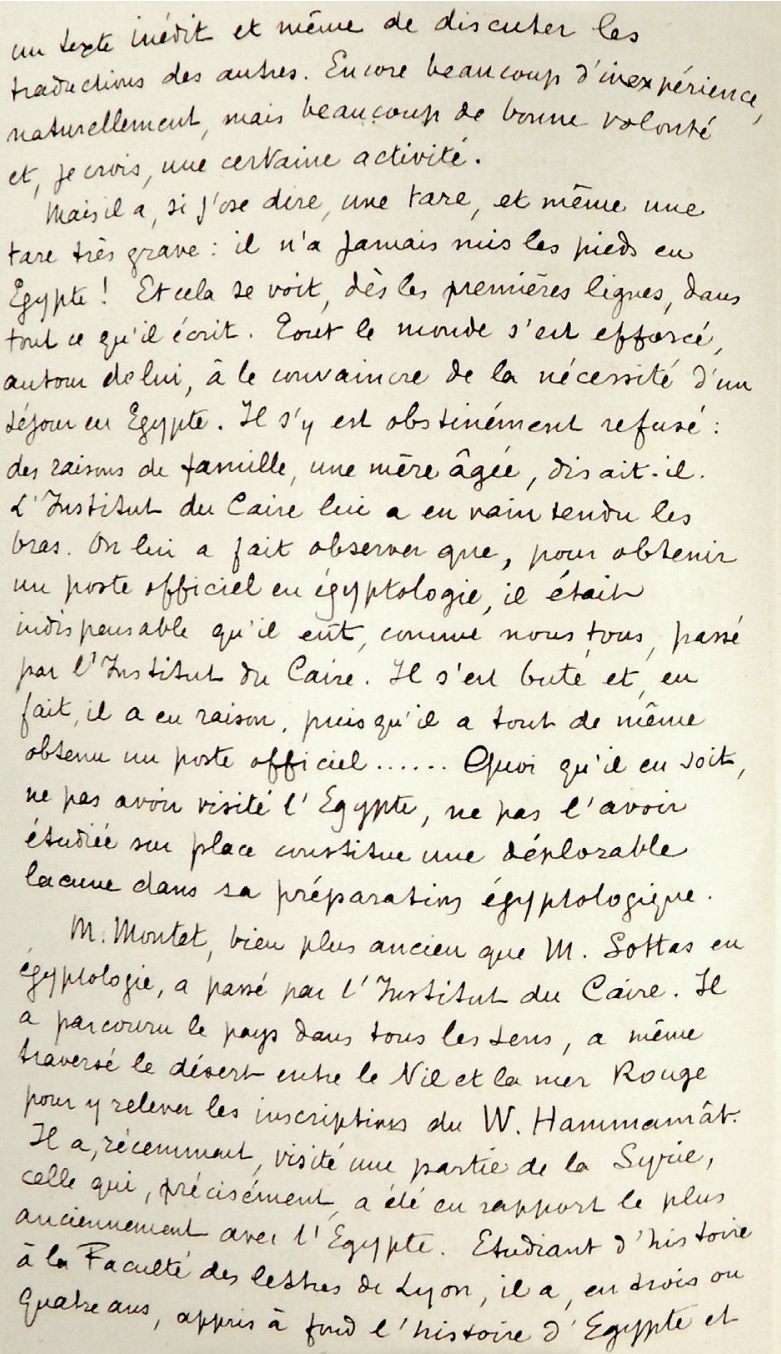 & 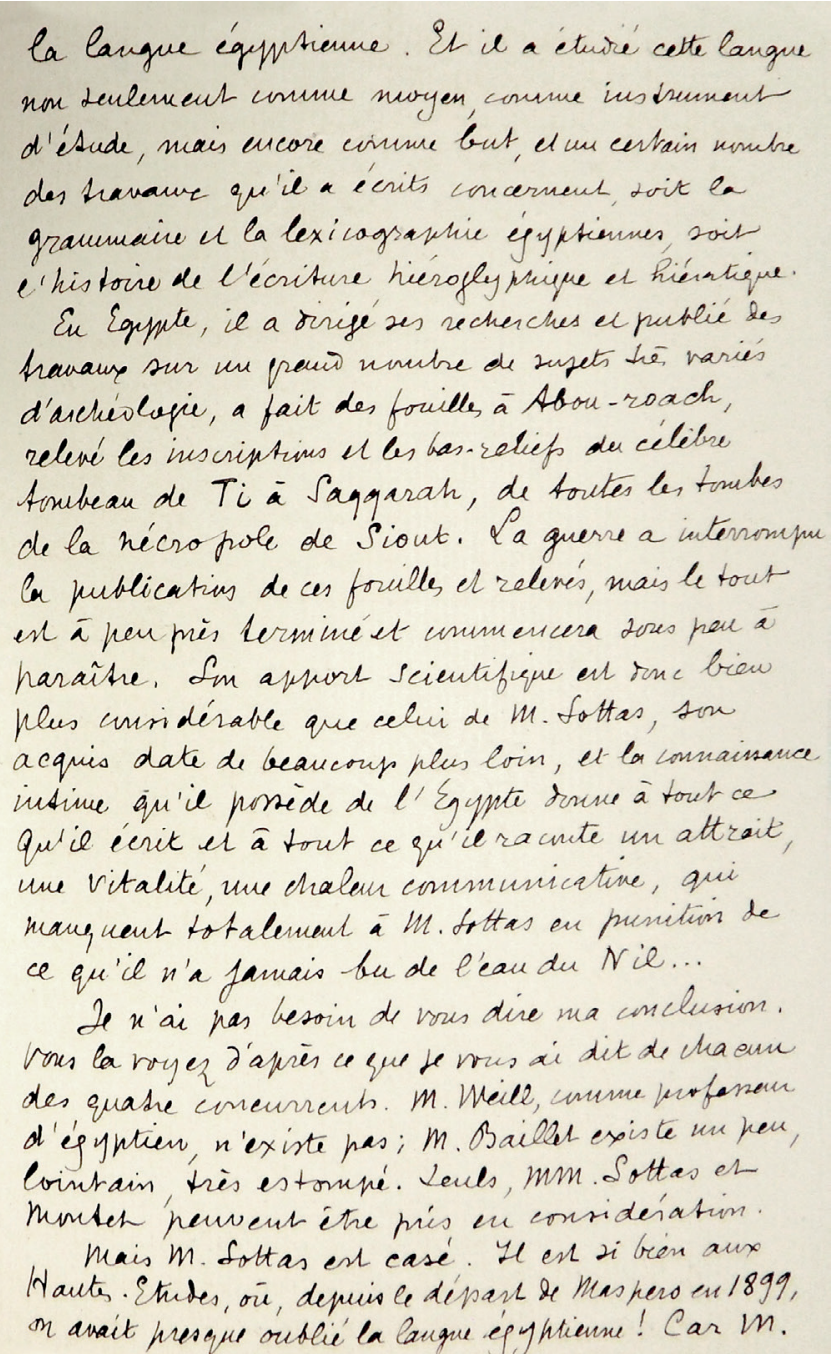 \\
\hline
\end{tabular}

Fig. 5 : ADBR 1007W 1251, lettre de V. Loret au recteur S. Charléty, page 6 et 7.

En plus des cours, Pierre Montet donne également des conférences dans le cadre de l'université publique [30] et participe aux « réunions du samedi » durant lesquelles les enseignants de la Faculté présentent tour à tour des comptes rendus critiques d'ouvrages parus récemment. Un résumé de ces séances paraît régulièrement dans le Bulletin de la Faculté des Lettres de Strasbourg [31].

[30] ADBR 1007W 1251, notice individuelle du Service de I'Instruction publique du 8 juin 1921.

[31] Lors de la séance du 22 janvier 1927, Pierre Montet a présenté les ouvrages de Georges Contenau, La civilisation phénicienne, Paris, 1928 ; Charles Kuentz, L'oie du Nil dans l'antique Égypte, Lyon, 1926 ; Wilhelm Spiegelberg, Demotische Grammatik, Heidelberg, 1925 ; Adolf Erman \& Hermann Grapow, Wörterbuch der aegyptischen Sprache, Berlin, 1926 (Bulletin de la Faculté des Lettres de Strasbourg, 1927, p. 180-181). Le 28 mars 1928, il propose un compte rendu de I'Egyptian Grammar d'Alan Gardiner, Oxford, 1927, et de I'Atlas zur Altägytischen Kulturgesichte, Leipzig, 1927, de Walter Wreszynski (Bulletin de la Faculté des
À partir de 1921, P. Montet entreprend à la demande de l'Académie des Inscriptions et Belles-Lettres, des fouilles au Liban, sur le site de Byblos, la mission effectuée en 1919 lui ayant permis de se forger une bonne connaissance du terrain. La campagne dure en moyenne 3 mois ; P. Montet doit donc chaque année demander l'autorisation de s'absenter de l'université, comme le montrent plusieurs documents conservés dans son dossier personnel [32]. En 1923, il découvre

Lettres de Strasbourg, 1928, p. 222). Le 14 janvier 1933, $P$. Montet présente un résumé critique de La religion des anciens égyptiens de Kurthe Sethe. Une présentation par L. Buchholzer-Remy, S. Donnat, G. Ducoeur et I. Laboulais et une table-ronde avec O. Dumoulin, W. Feuerhahn, Ch. de Montlibert et $\mathrm{B}$. Müller sur ces réunions du samedi a eu lieu le 21 novembre 2019 à la Maison Interuniversitaire des Sciences de I'Homme-Alsace dans le cadre d'un colloque intitulé « L'Université de Strasbourg et la dialogue des disciplines. Des années 1920 aux pratiques contemporaines » (non vidi). [32] ADBR 1007W 1251, lettre de P. Montet au doyen Chr. Pfister, datée du 5 janvier 1924 ; lettre de P. Montet au doyen G. Maugain, datée du 16 avril 1928. 
quatre tombes inviolées de plusieurs rois de Byblos du deuxième millénaire avant notre ère. Cette trouvaille obtient un grand retentissement médiatique et une exposition est organisée au Louvre l'année suivante, afin d'y présenter des pièces provenant de plusieurs fouilles françaises au Liban dont les objets découverts par P. Montet sont les masterpieces [33]. Cette exposition est pour l'archéologue l'occasion de signaler que les fonds manquent pour poursuivre les recherches à Byblos. Malheureusement, cet appel ne fut pas ou peu entendu, puisque les fouilles sous la direction de l'égyptologue strasbourgeois s'arrêtent en 1924. La publication de ces travaux paraît en deux volumes en 1928 [34].

Sur le plan des publications, Pierre Montet termine durant ses premières années à Strasbourg plusieurs manuscrits, qui devaient initialement être tous publiés aux presses de I'Ifao, ces travaux ayant été commencés durant son pensionnat au Caire. Une lettre conservée dans son dossier strasbourgeois montre cependant que le changement de directeur de l'Ifao a entraîné le retard ou même l'annulation de l'impression de certains de ces ouvrages [35], qui avaient pourtant été acceptés par P. Lacau en 1914. Le remaniement d'une partie de ces manuscrits donne lieu à la thèse de P. Montet, Scènes de la vie privée des tombeaux égyptiens de l'Ancien Empire [36], qu'il soutient à Lyon en 1925.

Après avoir obtenu son doctorat, Pierre Montet devient maître de conférences en 1925 puis professeur sans chaire. Il est titularisé en 1935, avec le soutien de son collègue Paul Perdrizet, qui présente au doyen Gabriel Maugain un rapport élogieux sur ses travaux [37].

\section{LES FOUILLES DE TANIS ET LE REPLI À CLERMONT-FERRAND (1928-1939)}

En 1928, Pierre Montet entreprend du 25 avril à la fin mai sa première campagne de fouilles à Tanis, dans le Delta égyptien, à la demande du Ministre de I'Instruction publique [38]. Ce site, déjà en partie dégagé par Auguste Mariette, puis par Flinders Petrie à la fin du XIXe siècle, n'avait pas été complétement étudié. Après quatorze années sans travaux en Égypte, P. Montet retrouve donc le terrain. Ce projet débute au moment où I'Université de Strasbourg connaît une baisse importante de moyens financiers [39], et c'est probablement une des raisons qui pousse P. Montet à créer « La société des amis de Tanis », une association regroupant des particuliers intéressés par l'Égypte antique. Le premier membre d'honneur est le roi Fouad

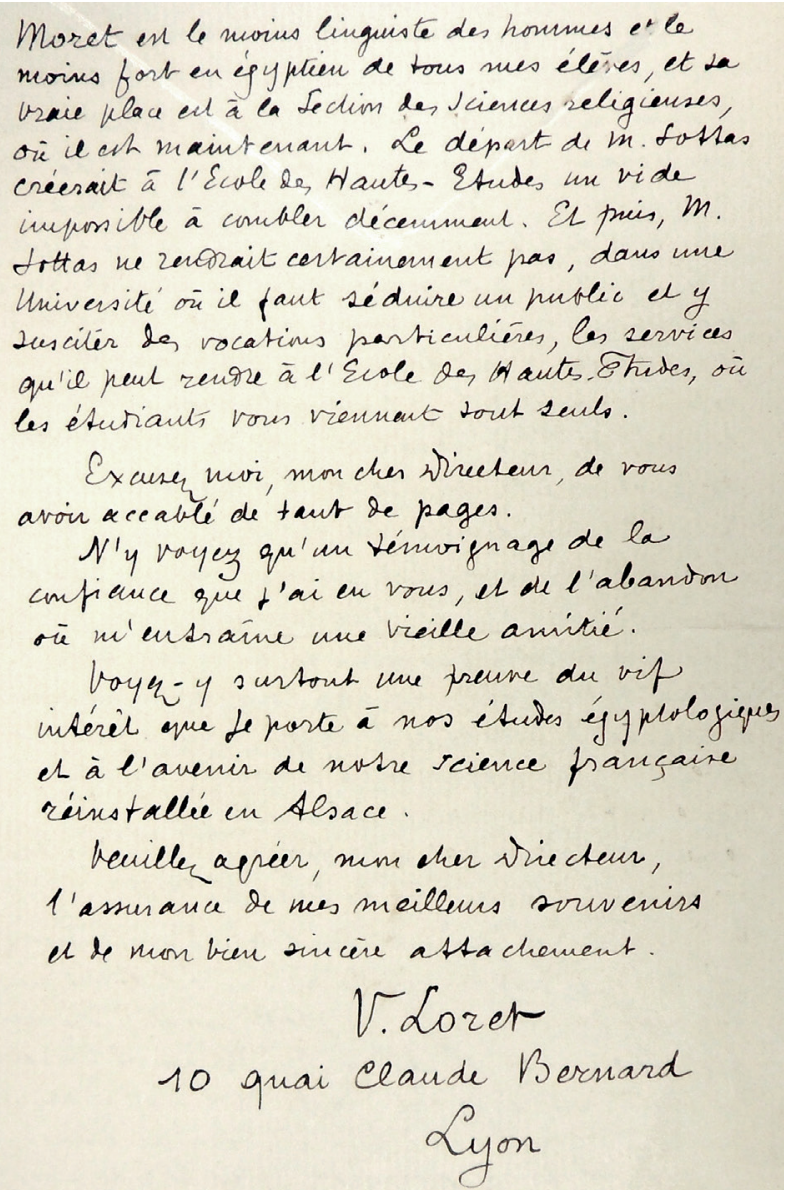

Fig. 6 :

ADBR 1007W 1251,

lettre de V. Loret au recteur S. Charléty, page 8.

[33] https://www.retronews.fr/journal/la-croix/22avril-1924/106/618027/2?from $=\% 2 F s e a r c h \% 23$ allTerms $\% 3$ Dbyblos\%2520montet $\% 26$ sort $\% 3$ Dscore $\% 26$ publishedBounds\%3Dfrom $\% 26$ indexedBounds $\% 3$ Dfrom $\%$ 26page\%3D1\%26searchIn\%3Dall\%26total\%3D145\&index $=16$

La Croix, 22 avril 1924 « Une exposition d'archéologie orientale au Musée du Louvre », J. Restoudière.

[34] MONTET 1928.

[35] ADBR 1007W 1251, lettre de P. Montet datée du 25 mai 1920, à S. Charléty. Voici la liste des ouvrages cités par P. Montet dans cette missive : «1. Les tombeaux de Siout et de Rifeh ; 2 . Deux tombeaux de la VIe dynastie à Kasr-el-sayad ; 3 . Tombeaux thébains ; 4. Une nécropole de la 1 ère dynastie : Abou Roach ; 5 et 6 . La décoration des tombes de l'Ancien Empire - Études sur la grammaire des textes contenus dans les tombes de l'Ancien Empire ». [36] MONTET 1924 et VANDIER 1966, p. 344.

[37] ADBR 1007W 1251, « Rapport présenté à M. le Doyen Maugain par M. Perdrizet sur la titularisation de M. Pierre Montet », non daté.

[38] Un arrêté du 5 avril 1928 charge officiellement P. Montet de cette mission (ADBR 1007W 1251).

[39] MüLLER 2018. 
$1^{\text {er }}$ d'Égypte, à qui sera par ailleurs décerné le titre de doctor honoris causa de I'Université de Strasbourg en 1932, sur proposition de P. Montet. Dans un rapport présenté en 1935 dans le cadre de l'avancement de carrière de l'égyptologue strasbourgeois [40], Paul Perdrizet signale que la société compte plus de 200 membres dispersés dans toute la France, soulignant ainsi l'intérêt que suscitent les travaux de l'égyptologue auprès du grand public.

De ces fouilles conduites chaque année sans interruption jusqu'au milieu de la Seconde Guerre mondiale, Pierre Montet rapporte une série d'objets qu'il partage entre les collections du Louvre (qui finance une partie de ses campagnes) et de l'Institut de Strasbourg [41]. L'inventaire rédigé par l'abbé Paul Bucher, collaborateur de P. Montet, ne permet pas de savoir exactement combien de pièces ont été ramenées de Tanis en Alsace (les provenances ne sont pas indiquées), mais il est certain qu'elles se comptent en centaines. Le colosse de Ramsès II [42], conservé au Palais universitaire, est I'un des plus beaux artefacts de cet ensemble. En plus de ces objets, P. Montet a laissé à I'Institut d'égyptologie une série de 900 plaques photographiques sur verre (positifs et négatifs), lesquelles donnent un aperçu très vivant du travail effectué par son équipe et ses dizaines de fouilleurs [43].

En 1939, à I'annonce de la guerre, I'Université de Strasbourg prend la décision de se replier en zone libre, à Clermont-Ferrand, où I'université locale accepte de partager ses bâtiments. Dès le début 1939, alors que P. Montet se trouve en Égypte et réalise la campagne de fouilles qui va le rendre célèbre, sa femme et ses filles organisent leur déménagement de Strasbourg à Paris. Les premières tombes de la nécropole royale de Tanis avaient été découvertes l'année précédente et Pierre Montet devait partir pour une mission de novembre 1939 à mars 1940. Le déroulement de la mission a été remis en question à cause des évènements internationaux, comme nous l'apprend une lettre du doyen au recteur André Terracher datée du 24 juin 1939, car le conseil de la Faculté des Lettres a débattu pour savoir si les circonstances permettaient que Pierre Montet parte en mission [44].
Ensuite, une demande d'autorisation d'absence a été transmise au Ministère de l'éducation nationale et au Ministère des affaires étrangères, qui ont tous deux donné leur accord [45]. Dans le but de convaincre les autorités de le laisser se rendre en Égypte, P. Montet propose de prendre lui-même en charge le salaire de son remplaçant à l'université, Alexandre Varille, afin de ne pas occasionner de dépense supplémentaire à son employeur [46]. Les archives de I'université ont conservé une transcription d'une lettre datée du 9 mars, envoyée par Étienne Drioton (directeur du service des Antiquités égyptiennes au Caire) au doyen de la Faculté des Lettres, annonçant la découverte de la tombe du pharaon Psousennès $1^{\mathrm{er}}:$ « (...) M. P. MONTET, professeur de votre université, vient de faire une découverte qui marquera dans les annales de la science : il a déblayé à SAN-el-HAGAR (Tanis), le caveau inviolé du roi Psousennès $1^{\mathrm{er}}$, qui renfermait un trésor de vases et de bijoux d'or de la plus haute qualité et de la plus grande importance pour l'étude de la civilisation égyptienne. Une salle spéciale du Musée du Caire est en préparation pour les recevoir, qui portera le nom de «Salle de Tanis (Fouilles de I'Université de STRASBOURG) ». (...) Étant donné l'insécurité des temps et le danger qu'il y aurait à laisser, même sous garde militaire, un trésor présumé dans un endroit aussi reculé que SAN-el-HAGAR, le vœu unanime de Sa Majesté le Roi, du Gouvernement Égyptien et de l'Égypte tout entière est que M. MONTET puisse prolonger le temps de sa mission afin de procéder d'urgence aux travaux d'ouverture et d'exploration de cette seconde chambre » [47].

La découverte des tombeaux de Tanis apporte une certaine célébrité à Pierre Montet : ses cours, qui à ses débuts, n'étaient suivis que par un nombre réduit d'étudiants, font salle comble. Dans une note adressée au doyen de la Faculté des Lettres de Strasbourg [48], le doyen de la Faculté des Lettres de Clermont-Ferrand demande à son homologue alsacien de bien veiller à ce que la salle dans laquelle P. Montet donne cours soit équipée de haut-parleurs. Des étudiants se sont en effet plaints de ne pas pouvoir entendre correctement les propos de l'égyptologue depuis le fond
[40] ADBR 1007W 1251, rapport cité plus haut.

[41] Au sujet de la réglementation du partage des fouilles en Egypte, voir notamment KHATER 1960 et REID 2015. [42] HARTENSTEIN 2015 ; Colin \& HARTENSTEIN 2016a.

[43] HaRTENSTEIn 2015 ; Colin \& HaRTENSTEIn 2016b.

[44] ADBR op. cit., lettre du recteur A. Terracher au conseiller d'État, datée du 24 juin 1939.

[45] ADBR op. cit., lettre du conseiller d'État au recteur A. Terracher, datée du 25 juillet 1939.

[46] ADBR op. cit., lettre du recteur A. Terracher au conseiller d'État, datée du 24 juin 1939. A. Terracher précise que le remplacement devait avoir lieu du $1^{\mathrm{er}}$ novembre au $1^{\mathrm{er}}$ mars. Les archives de l'université que j'ai consultées ne m'ont pas permis de savoir si A. Varille a bien effectué ce remplacement. [47] ADBR 1007W 1251. Dans ce même document contenant la transcription du télégramme, le vice-recteur de I'université précise qu'il va transmettre son accord à la demande de prolongation du séjour de P. Montet. L'égyptologue rentre en France en mai 1940.

[48] ADBR 154 AL 14, lettre du doyen Waltz du 19 novembre 1940 au doyen G. Maugain. 
de l'amphithéâtre. Les fouilles de Tanis valent également à P. Montet les honneurs de nombreux journaux. Cependant, les conditions de travail dans les locaux de l'université clermontoise ne sont pas toujours optimales : dans une lettre datée du 20 janvier 1942 [49], P. Montet rapporte au doyen Maugain que I'administration clermontoise lui demande de quitter la petite salle de travail qu'il partage avec deux collègues (Baulig et Cavaignac), dans laquelle se trouvent les livres de la bibliothèque d'égyptologie et ses documents de travail et dans laquelle il donne parfois cours. Il se tourne donc vers son supérieur afin de trouver du soutien, car il estime nécessaire que les ouvrages soient accessibles aux étudiants d'égyptologie.

[49] ADBR 154 AL 14, lettre de P. Montet au doyen G. Maugain.
Les activités archéologiques de Pierre Montet, soulignées par Victor Loret dans sa lettre de recommandation en 1919, sont donc en grande partie à I'origine de la renommée du savant dans le milieu des spécialistes, et même au-delà du cercle universitaire. L'objectif fixé par la nouvelle université strasbourgeoise, productivité scientifique et rayonnement international, est donc pleinement atteint dans le domaine de l'égyptologie. Après la fin de la Seconde Guerre mondiale, Pierre Montet retourne effectuer une dernière campagne à Tanis, en 1947, sous la bannière strasbourgeoise, avant d'être nommé Professeur au Collège de France en 1948. Le remplacement de ses cours sera assuré pendant plusieurs années par son ami I'abbé Bucher. L'œuvre de Pierre Montet a durablement marqué la ville et I'université alsaciennes : une plaque signale ses réalisations sur la façade du 1 rue Arbogast, où il vécut une partie de sa période strasbourgeoise avec sa femme et ses filles, et une rue du campus de l'Esplanade a été nommée en son honneur.

\section{REMERCIEMENTS}

Je remercie avec plaisir les deux experts anonymes pour leurs remarques en vue de l'amélioration du manuscrit. 


\section{ADBR $10071251 \mathrm{~W}$}

Lyon, 9 sept(embre) 1919

Mon cher Directeur,

Je dois aller sous peu passer quelques semaines à Paris, et j'espère avoir le plaisir de vous y voir. En attendant, je voudrais vous entretenir de diverses questions égyptologiques dont la solution doit être toute prochaine.

Tout d'abord, la question Kuentz. Vous vous souvenez de ce jeune égyptologue au sujet duquel je vous ai écrit à propos de son admission à l'Institut du Caire. Divers malentendus, - causés uniquement par le torpillage de la plus grande partie des lettres qu'ils échangeaient, - s'étaient élevés entre M. Foucart et lui. Depuis, on a pu s'expliquer, et tout est arrangé. M. Kuentz a du reste grandement mérité bienveillance et sympathie : demeuré seulement sept mois à l'École normale et y travaillant avec une ardeur acharnée, il en est sorti, il y a quelques semaines, premier à l'agrégation de langues classiques. M. Montet quittant I'Institut du Caire et une place de pensionnaire se trouvant ainsi libre pour novembre prochain, M. Foucart m'a appris sa décision de présenter Kuentz en octobre et il l'engage même à se munir dès maintenant d'un passeport et d'une place sur le premier bateau de novembre.

M. Kuentz est certainement le plus brillamment doué de tous les élèves que j'ai eus jusqu'ici et je ne doute pas qu'il n'arrive très vite à être un de nos savants les plus remarquables. Les trois ou quatre travaux qu'il a déjà publiés ont attiré sur son nom la plus flatteuse attention de nos collègues égyptologues, français et étrangers.

Je viens donc, mon cher Directeur, comme conclusion à cette première question, vous demander de vouloir bien accueillir favorablement la proposition qui vous sera faite par $\mathrm{M}$. Foucart, lors de la prochaine réunion du comité, de nommer $\mathrm{M}$. Kuentz pensionnaire de I'Institut français du Caire. Vous n'aurez, nous n'aurons tous, j'en suis sûr, qu'à vous louer de ce choix.

Seconde question : l'égyptologie à l'Université de Strasbourg. Cette université possédant un très intéressant musée égyptologique, et dans sa bibliothèque, un fonds très riche de livres d'égyptologie, il eut été infiniment regrettable qu'on y supprimât l'enseignement de l'égyptologie, surtout après la disparition de la chaire d'égyptologie d'Alger par la suite de la mort de M. Lefébure. Mais cette suppression, m'annonce-t-on, n'est plus à craindre, et nous ne saurions vous en dire toute notre reconnaissance.

Quatre candidats, si je suis bien informé, se présentent pour l'enseignement de l'égyptologie à Strasbourg : MM. Raymond Weill, Jules Baillet, Sottas et Montet. Voulez-vous me permettre, à titre - hélas ! - de plus ancien égyptologue français, de vous fournir des renseignements précis et sincères sur la valeur de chacun de ces quatre candidats? Il ne doit pas vous paraître inutile, j'en suis certain, d'être renseigné exactement par un homme du métier et par un ancien ami qui peut se permettre de vous parler à cœur ouvert.

M. Raymond Weill, ancien capitaine du Génie, a de très grandes qualités comme fouilleur et leveur de plans. Sous ce rapport, il n'a de rival que M. de Morgan, et tous les travaux qu'il a publiés dans cet ordre d'idées lui ont valu l'admiration générale. Il a passé son doctorat avec une thèse sur un sujet d'histoire et d'archéologie qu'il a traité de façon assez terne et sans y apporter beaucoup de nouveau ; mais cette thèse est bien ordonnée et richement documentée. Son grand, son terrible défaut est celui-ci : il ne connaît pas un traître mot d'égyptien ! La grammaire égyptienne lui est totalement inconnue. Et le pire est qu'il ne se doute même pas qu'il ne sait pas l'égyptien. De sorte qu'il publie, en toute innocence, les traductions les plus saugrenues et les plus inimaginables, qui font se pâmer de rire, ou d'indignation, selon les caractères, ceux qui lisent ses interprétations de textes hiéroglyphiques. Jugez-en par ces quelques lignes écrites, à propos d'un travail de lui paru en 1912 («Décrets royaux de Koptos »), par un des meilleurs égyptologues anglais, très gentleman d'ailleurs et très ami de la France, M. Alan H. Gardiner : « It is regrettable that the broad principles of Egyptian grammar, now so fully and certainly established, should still be ignored by so active and industrious an Egyptologist as M. Weill. It is difficult to imagine how, ex.gr. the renderings « ... » could be arrived at, and a similar guessing at the sense is everywhere apparent. As examples of particularly crass grammatical blunders I will only quote the translation «... », etc, etc (Proceedings of the Society of Biblical Archaeology, vol. XXXIV, London, 1912, p. 265).

M. Lacau songerait, m'a-t-on dit, à faire entrer M. Weill au Service des Antiquités de l'Égypte, comme successeur de Legrain à la direction des fouilles de Thèbes. Là serait sa vraie place et il y rendrait d'immenses services. Mais lui confier l'enseignement de l'égyptologie à Strasbourg ? L'égyptologie allemande en trépignerait de joie.

M. Jules Baillet est le fils d'un égyptologue, Auguste Baillet, dont les œuvres, qui forment deux assez gros volumes, ont été publiées en collaboration par le père et le fils et sont quelquefois, par les esprits superficiels, confondues avec les œuvres du fils. Celles-ci sont peu nombreuses : trois ou quatre articles (exactement comme M. Kuentz) et deux thèses de doctorat, dont la principale a 810 pages ! M. J. Baillet est depuis longtemps professeur de lycée. Malgré ses accaparantes occupations professionnelles, il a trouvé le moyen de se tenir étonnamment au courant de toute la littérature égyptologique. Peu des nôtres sont, sous ce rapport, aussi bien outillés que lui, et l'index bibliographique de sa thèse (150 p. à 2 col. très serrées) rend journellement les plus grands services à tous. 
Mais, avec toutes ces qualités, M. Baillet donne dans ses travaux une étrange impression. L'appareil critique est moderne ; les idées semblent dater de 1830 ! Comment et pourquoi ? Je n'ai pu encore me l'expliquer de façon satisfaisante. Je crois que M. Baillet, qui a su si bien se tenir au courant des recherches des autres, n'a pas eu le temps de se livrer suffisamment à des recherches personnelles. D'ailleurs, la linguistique orientale n'est pas son fort, loin de là, et il n'a pas dû lire et traduire beaucoup de textes égyptiens, ni surtout avec toute la minutie et la précision qu'il faut y mettre pour en tirer des idées nouvelles. De là, sans doute, ce quelque chose de grisâtre, d'impersonnel, de vieillot qui se dégage de la lecture de ses écrits. En somme, il n'a, à aucun degré, la mentalité d'un professeur d'enseignement supérieur, et, très vraisemblablement, ne l'acquerra jamais.

M. Sottas est, relativement, un débutant ; mais, il faut le reconnaître, un débutant qui promet. Il a une grande qualité, assez rare en France : il connaît la grammaire égyptienne. Non pas qu'il soit grammairien par goût. Je ne crois pas qu'il écrira jamais rien sur la grammaire. Mais il a compris que, pour faire de l'égyptologie, il faut avant tout apprendre l'égyptien, et il s'en est donné la peine. Il est capable de traduire un texte inédit et même de discuter les traductions des autres. Encore beaucoup d'inexpérience, naturellement, mais beaucoup de bonne volonté et, je crois, une certaine activité.

Mais il a, si j'ose dire, une tare, et même une tare très grave : il n'a jamais mis les pieds en Égypte ! Et cela se voit, dès les premières lignes, dans tout ce qu'il écrit. Tout le monde s'est efforcé, autour de lui, à le convaincre de la nécessité d'un séjour en Égypte. Il s'y est obstinément refusé : des raisons de famille, une mère âgée, disait-il. L'Institut du Caire lui a en vain tendu les bras. On lui a fait observer que, pour obtenir un poste officiel en égyptologie, il était indispensable qu'il eût, comme nous tous, passé par I'Institut du Caire. Il s'est buté et, en fait, il a eu raison, puisqu'il a tout de même obtenu un poste officiel ...... Quoi qu'il en soit, ne pas avoir visité l'Égypte, ne pas l'avoir étudiée sur place constitue une déplorable lacune dans sa préparation égyptologique.

M. Montet, bien plus ancien que M. Sottas en égyptologie, a passé par l'Institut du Caire. Il a parcouru le pays dans tous les sens, a même traversé le désert entre le Nil et la mer Rouge pour y relever les inscriptions du W. Hammamât. Il a, récemment, visité une partie de la Syrie, celle qui, précisément, a été en rapport le plus anciennement avec l'Égypte. Étudiant d'histoire à la Faculté des lettres de Lyon, il a, en trois ou quatre ans, appris à fond l'histoire d'Égypte et la langue égyptienne. Et il a étudié cette langue non seulement comme moyen, comme instrument d'étude, mais encore comme but, et un certain nombre des travaux qu'il a écrits concernent soit la grammaire et la lexicographie égyptiennes, soit I'histoire de l'écriture hiéroglyphique et hiératique.

En Égypte, il a dirigé ses recherches et publié des travaux sur un grand nombre de sujets très variés d'archéologie, a fait des fouilles à Abou-roach, relevé les inscriptions et les bas-reliefs du célèbre tombeau de Ti à Saqqarah, de toutes les tombes de la nécropole de Siout. La guerre a interrompu la publication de ces fouilles et relevés, mais le tout est à peu près terminé et commencera sous peu à paraître. Son apport scientifique est donc bien plus considérable que celui de $M$. Sottas, son acquis date de beaucoup plus loin, et la connaissance intime qu'il possède de l'Égypte donne à tout ce qu'il écrit et à tout ce qu'il raconte un attrait, une vitalité, une chaleur communicative, qui manquent totalement à M. Sottas en punition de ce qu'il n'a jamais bu de l'eau du Nil...

Je n'ai pas besoin de vous dire ma conclusion, vous la voyez d'après ce que je vous ai dit de chacun des quatre concurrents. M. Weill, comme professeur d'égyptien, n'existe pas ; M. Baillet existe un peu, lointain, très estompé. Seuls, MM. Sottas et Montet peuvent être pris en considération.

Mais M. Sottas est casé. Il est si bien aux Hautes-Études, où, depuis le départ de Maspéro en 1899, on avait presque oublié la langue égyptienne ! Car M. Moret est le moins linguiste des hommes et le moins fort en égyptien de tous mes élèves, et sa vraie place est à la Section des Sciences religieuses, où il est maintenant. Le départ de M. Sottas créerait à l'École des Hautes-Études un vide impossible à combler décemment. Et puis, M. Sottas ne rendrait certainement pas, dans une université où il faut séduire un public et y susciter des vocations particulières, les services qu'il peut rendre à l'École des Hautes-Études, où les étudiants vous viennent tout seuls.

Excusez-moi, mon cher Directeur, de vous avoir accablé de tant de pages.

N’y voyez qu'un témoignage de la confiance que j'ai en vous, et de l'abandon où m'entraîne une vieille amitié.

Voyez-y surtout une preuve du vif intérêt que je porte à nos études égyptologiques et à l'avenir de notre science française réinstallée en Alsace.

Veuillez agréer, mon cher Directeur, l'assurance de mes meilleurs souvenirs et de mon bien sincère attachement.

V. Loret

10 Quai Claude Bernard

Lyon 
Liste des documents cités et conservés aux Archives Départementales du Bas-Rhin

\begin{tabular}{|c|c|c|c|c|}
\hline Cote & Date & Émetteur & Destinataire & Contenu \\
\hline $1161 W 73$ & $\begin{array}{l}27 \text { décembre } \\
1918\end{array}$ & W. Spiegelberg & & $\begin{array}{l}\text { État des lieux des collections de } \\
\text { l'Institut d'égyptologie }\end{array}$ \\
\hline $1007 W 1251$ & 22 mars 1919 & P. Montet & $\begin{array}{l}\text { S. Charléty } \\
\text { (recteur) }\end{array}$ & Lettre de candidature \\
\hline $1007 W 1251$ & 6 août 1919 & V. Loret & $\begin{array}{l}\text { Chr. Pfister } \\
\text { (doyen de la } \\
\text { Faculté des } \\
\text { Lettres) }\end{array}$ & $\begin{array}{l}\text { Demande de renseignements } \\
\text { concernant le destinataire à qui la } \\
\text { lettre de recommandation pour P. } \\
\text { Montet doit être envoyée }\end{array}$ \\
\hline $1007 W 1251$ & $\begin{array}{l}9 \text { septembre } \\
1919\end{array}$ & P. Montet & $\begin{array}{l}\text { S. Charléty } \\
\text { (recteur) }\end{array}$ & Deuxième lettre de candidature \\
\hline 1007W 1251 & $\begin{array}{l}10 \text { septembre } \\
1919\end{array}$ & P. Montet & $\begin{array}{l}\text { S. Charléty } \\
\text { (recteur) }\end{array}$ & Troisième lettre de candidature \\
\hline 1007W 1251 & $\begin{array}{l}19 \text { septembre } \\
1919\end{array}$ & V. Loret & $\begin{array}{l}\text { S. Charléty } \\
\text { (recteur) }\end{array}$ & $\begin{array}{l}\text { Lettre de recommandation pour } \mathrm{P} \text {. } \\
\text { Montet }\end{array}$ \\
\hline 1007W 1251 & 17 mars 1920 & P. Montet & & $\begin{array}{l}\text { Notice individuelle du Service de } \\
\text { I'Instruction publique }\end{array}$ \\
\hline 1007W 1251 & 25 mai 1920 & P. Montet & $\begin{array}{l}\text { S. Charléty } \\
\text { (recteur) }\end{array}$ & $\begin{array}{l}\text { P. Montet demande de l'aide au } \\
\text { recteur car plusieurs ouvrages qu'il } \\
\text { a rédigés devaient être publiés par } \\
\text { les presses de l'Ifao }\end{array}$ \\
\hline 1007W 1251 & 8 juin 1921 & P. Montet & & $\begin{array}{l}\text { Notice individuelle du Service de } \\
\text { I'Instruction publique }\end{array}$ \\
\hline 1007W 1251 & 15 juin 1923 & P. Montet & $\begin{array}{l}\text { Chr. Pfister } \\
\text { (doyen de la } \\
\text { Faculté des } \\
\text { Lettres) }\end{array}$ & $\begin{array}{l}\text { Lettre de P. Montet expliquant I'or- } \\
\text { ganisation de ses cours }\end{array}$ \\
\hline 1007W 1251 & $\begin{array}{l}5 \text { janvier } \\
1924\end{array}$ & P. Montet & & $\begin{array}{l}\text { P. Montet demande I'autorisation de } \\
\text { s'absenter pour sa mission au Liban }\end{array}$ \\
\hline 1007W 1251 & 5 avril 1928 & $\begin{array}{l}\text { Conseiller d'État, } \\
\text { directeur général } \\
\text { des services d'Al- } \\
\text { sace et de Lorraine }\end{array}$ & $\begin{array}{l}\text { Recteur } \\
\text { d'Académie }\end{array}$ & $\begin{array}{c}\text { Arrêté ministériel qui charge P. } \\
\text { Montet d'effectuer des fouilles à } \\
\text { Tanis }\end{array}$ \\
\hline 1007W 1251 & 16 avril 1928 & P. Montet & $\begin{array}{l}\text { Chr. Pfister (doyen } \\
\text { de la Faculté des } \\
\text { Lettres) }\end{array}$ & $\begin{array}{l}\text { P. Montet demande l'autorisation de } \\
\text { s'absenter pour sa mission à Tanis }\end{array}$ \\
\hline 1007W 1251 & 24 juin 1939 & $\begin{array}{l}\text { A. Terracher } \\
\text { (recteur) }\end{array}$ & $\begin{array}{l}\text { Conseiller d'État, } \\
\text { directeur général } \\
\text { des services d'Al- } \\
\text { sace et de Lorraine }\end{array}$ & $\begin{array}{l}\text { A. Terracher demande l'autorisation } \\
\text { pour P. Montet de s'absenter pour } \\
\text { les fouilles de Tanis }\end{array}$ \\
\hline 1007W 1251 & $\begin{array}{l}25 \text { juillet } \\
1939\end{array}$ & $\begin{array}{l}\text { Conseiller d'État, } \\
\text { directeur général } \\
\text { des services d'Al- } \\
\text { sace et de Lorraine }\end{array}$ & A. Terracher & $\begin{array}{l}\text { Document autorisant P. Montet à } \\
\text { partir en fouilles à Tanis }\end{array}$ \\
\hline $1007 W 1251$ & 2 avril 1940 & $\begin{array}{l}\text { A. Terracher } \\
\text { (recteur) }\end{array}$ & $\begin{array}{l}\text { G. Maugain } \\
\text { (doyen de la } \\
\text { Faculté des } \\
\text { Lettres) }\end{array}$ & $\begin{array}{c}\text { Courrier retranscrivant le télé- } \\
\text { gramme envoyé par É. Drioton } \\
\text { concernant les découvertes des } \\
\text { tombes royales de Tanis et concer- } \\
\text { nant la suite donnée par l'université } \\
\text { à la demande de prolongation de la } \\
\text { mission de P. Montet. }\end{array}$ \\
\hline
\end{tabular}




\begin{tabular}{|c|c|c|c|c|}
\hline ADBR 154 AL 14 & $\begin{array}{c}19 \text { novembre } \\
1940\end{array}$ & $\begin{array}{c}\text { Waltz } \\
\text { (doyen de la Faculté } \\
\text { des Lettres de } \\
\text { Clermont-Ferrand) }\end{array}$ & $\begin{array}{c}\text { G. Maugain } \\
\text { (doyen de la } \\
\text { Faculté des } \\
\text { Lettres) }\end{array}$ & $\begin{array}{c}\text { Le doyen Waltz demande à G. } \\
\text { Maugain de veiller à ce que la salle } \\
\text { dans laquelle P. Montet fait cours } \\
\text { soit équipée de haut-parleurs. }\end{array}$ \\
\hline ADBR 154 AL 14 & $\begin{array}{c}20 \text { janvier } \\
1942\end{array}$ & P. Montet & $\begin{array}{c}\text { G. Maugain } \\
\text { (doyen de la } \\
\text { Faculté des } \\
\text { Lettres) }\end{array}$ & $\begin{array}{c}\text { P. Montet signale que I'administra- } \\
\text { tion de I'Université de Clermont- } \\
\text { Ferrand lui demande de vider } \\
\text { la salle qu'il occupe pour y faire } \\
\text { quelques cours, travailler et entre- } \\
\text { poser des ouvrages }\end{array}$ \\
\hline 1007 W 1251 & Sans date & P. Perdrizet & $\begin{array}{c}\text { G. Maugain } \\
\text { (doyen de la } \\
\text { Faculté des } \\
\text { Lettres) }\end{array}$ & $\begin{array}{c}\text { « Rapport présenté à M. le Doyen } \\
\text { Maugain par M. Perdrizet sur la } \\
\text { titularisation de M. Pierre Montet 》 }\end{array}$ \\
\hline
\end{tabular}

BIBLIOGRAPHIE

Colin, Frédéric, 2010, "Comment la création d'une "bibliothèque de papyrus" à Strasbourg compensa la perte des manuscrits précieux brûlés dans le siège de $1870 »$, Revue de la BNU 2, p. 24-47.

Colin, Frédéric \& HARTenstern, Cassandre, 2016a, « Eine kolossale Sitzfigur Ramses'II », dans Lars Petersen (dir.) Ramses, Göttlicher Herrscher am Nil, Catalogue d'exposition, Badisches Landesmuseum à Karlsruhe, Petersberg, p. 54-56.

Colin, Frédéric \& HARTenstein, Cassandre, 2016b, « Le projet photographique de W. Spiegelberg et la collection de plaques de verre de l'Institut d'égyptologie de Strasbourg (Spiegelberg-Montet) », communication dans le cadre du colloque Plaques photographiques : fabrication et diffusion du savoir, organisé à I'Université Strasbourg par Denise Borlée et Hervé Doucet, 16 mars 2016, https://www.canal-u.tv/video/uds/le projet photographique de w spiegelberg et la collection de plaques de verre de I institut d egyptologie de strasbourg spiegelberg montet.21467, consulté le 20 décembre 2020.

Colin, Frédéric \& Hartenstein, Cassandre, 2017, « Collectionner les sources du savoir : les fonds égyptologiques 》, dans Joëlle Pijaudier-Cabot \& Roland Recht (éd.), Laboratoire d'Europe, Strasbourg 1880-1930, Strasbourg, p. $258-269$.

Gertzen, Thomas L., 2016, « Giant of Egyptology, 33rd of a series: Wilhelm Leeser Spiegelberg (1870-1930) 》, KMT A Modern Journal of Ancient Egypt 27, p. 70-75.

Hartenstein, Cassandre, 2015, «Le fonds Montet et la statue “maussade" de Ramsès II », Archimède : archéologie et histoire ancienne 2, p. 41-50.

DOI : 10.47245/archimede.0002.ds1.04.

Hartenstein, Cassandre, 2017, « Pierre Montet », dans Roland Recht \& Jean-Claude Richez (éd.), Dictionnaire culturel de Strasbourg 1880-1930, Strasbourg, p. 351.

Khater, Antoine, 1960, Le régime juridique des fouilles en Égypte, Le Caire (Recherches d'archéologie, de philologie et d'histoire 12).

LoRentz, Claude, 2000, Les fonds anciens de I'Université Marc Bloch de Strasbourg : historique, essai d'évaluation et situation générale, mémoire d'étude sous la direction de Dominique Varry,

URL : http://enssibal.enssib.fr/bibliotheque/documents/dcb/lorentz.pdf

Montet, Pierre, 1924, Scènes de la vie privée dans les tombeaux égyptiens de l'Ancien Empire, Strasbourg.

Montet, Pierre, 1926, « L'institut égyptologique », Bulletin de la faculté des lettres de Strasbourg 5, p. 171-174.

MüLLeR, Bertrand, 2018, «L'Université de Strasbourg dans l'immédiat après-guerre (1919-1925) 》, Revue d'histoire des sciences humaines [En ligne], 33 | 2018, mis en ligne le 22 mars 2019, consulté le 02 décembre 2020,

URL : http://journals.openedition.org/rhsh/1132.

DOI : $\underline{10.4000 / \text { rhsh.1132 }}$ 
Olivier-UtaRd, Françoise, 2010, « L'Université de Strasbourg de 1919 à 1939 : s'ouvrir à l'international mais ignorer I'Allemagne », Les Cahiers de Framespa [En ligne], 6 | 2010, mis en ligne le 01 décembre 2010, consulté le 02 décembre 2020. URL : http://journals.openedition.org/framespa/515.

DOI : $10.4000 /$ framespa. 515

OlivieR-UTARD, Françoise, 2015, Une université idéale ? Histoire de I'Université de Strasbourg de 1919 à 1939, Strasbourg. ReID, Donald, 2015, Contesting Antiquities in Egypt, Cairo.

Robert, Bruno, 2018, « Il y a 100 ans, Pierre Montet dans la Grande guerre », Cahier de la Société française des Fouilles de Tanis 11, p. 1-21.

SaUneron, Serge, 1968, « Pierre Montet (1885-1966)», Kémi 18, p. 9-15.

SpifgelberG, Wilhelm, 1902, «Ein neuer astronomischer Text auf einem demotischen Ostrakon », Orientalische Literaturzeitung 5, col. 223-225.

Spiegelberg, Wilhelm, 1904, « Varia », Recueil de travaux relatifs à la philologie et à l'archéologie égyptiennes et assyriennes 26, p. 143-154.

Spiegelberg, Wilhelm, 1906, « Ein Denkstein auf den Tod einer heiligen Isiskuh », Zeitschrift für ägyptische Sprache und Altertumskunde 43, p. 129-135.

Spiegelberg, Wilhelm, 1908, « Demotische Miscellen », RecTrav 30, p. 141-159.

Spiegelberg, Wilhelm, 1908-1909, « Neue demotischen Inschriften », Zeitschrift für ägyptische Sprache und Altertumskunde 45, p. 97-102.

Spiegelberg, Wilhelm, 1909, Ausgewählte Kunst-Denkmäler der aegyptischen Sammlung der Kaiser Wilhelms-Universität Strassburg, Strassburg.

Spiegelberg, Wilhelm, 1910, «Die ägyptischen Namen und Zeichen der Tierkreisbilder in demotischer Schrift 》, Zeitschrift für ägyptische Sprache und Altertumskunde 48, p. 146-151.

SPIegelberG, Wilhelm, 1912, « Ein demotischer Grabstein der römischen Kaiserzeit », Zeitschrift für ägyptische Sprache und Altertumskunde 50, p. 43-44.

Spiegelberg, Wilhelm, 1914, « Neue Denkmäler des Parthenios, des Verwalters der Isis von Koptos », Zeitschrift für ägyptische Sprache und Altertumskunde 51, p. 75-88.

Spiegelberg, Wilhelm, 1917, «Demotischen Miszellen », Zeitschrift für ägyptische Sprache und Altertumskunde 53, p. $116-129$.

Spiegelberg, Wilhelm, 1918a, «Eine Bronzestatuette des Amon », Zeitschrift für ägyptische Sprache und Altertumskunde 54, p. 74-76.

Spiegelberg, Wilhelm, 1918b, « Ein Heiligum des Gottes Chnum von Elephantine in der thebanischen Totenstadt », Zeitschrift für ägyptische Sprache und Altertumskunde 54, p. 64-67.

SpIegelberg, Wilhelm, 1920, « Neue Schenkungstelen über Landstiftungen an Tempel », Zeitschrift für ägyptische Sprache und Altertumskunde 56, p. 55-60.

Stracmans, Maurice, 1951, « Nécrologie : Raymond Weill (1874-1950) », Chronique d'Égypte 26 (51), p. $115-119$.

VANDIER, Jacques, 1951, «Raymond Weill (1874-1950)», Revue d'Égyptologie 8, p. i-vi.

VANDier, Jacques, 1966, «Éloge funèbre de M. Pierre Montet, membre de l'Académie », Comptes rendus des séances de I'Académie des Inscriptions et Belles-Lettres 100/6, p. 342-348.

Voss, Suzanne \& GerTzen, Thomas L., 2020, « German Egyptology (1882-1914) », UCLA Encyclopedia of Egyptology. WALle, Baudoin van de, 1967, « Pierre Montet (1885-1966)», Chronique d'Égypte 42 (83), p. 124-125.

Werll, Raymond, 1929, « Henri Sottas », Revue de l'Égypte ancienne 2, p. 121-127.

Wolff, Étienne, 1967, « Pierre Montet (1885-1966)», Revue d'Égyptologie 19, p. 7-9. 